\section{Applying RT deadlines to discrimination reaction time*}

\author{
S. W. LINK† \\ McMaster University, Hamilton, Ont., Canada
}

Five Ss discriminating differences between a fixed standard line length and five comparison line lengths performed under three randomly presented RT deadlines. Arralyses of response measures, conditional upon the RT deadline of a preceding trial, showed that on a trial-to-trial basis, $S$ could shift both accuracy and RT performance to meet the demands of a new RT deadline. There was no influence of the RT deadline used on Trial $n-1$ on performance on Trial $n$.

Placing RT deadlines on a discrimination RT task provides a method for interrupting an ongoing process at E-contrived times. The method has been used by numerous Es (Fitts, 1966; Pachella \& Pew, 1968; Yellott, 1967, 1971; Link \& Tindall, 1971) in order to change response accuracy in both discrimination and choice RT experiments. Yellott (1971) has suggested that the effect of an RT deadline in CRT experiments is to alter a mixture of fast guess and choice RT distributions. On the other hand, Link \& Tindall (1971) showed that in discrimination $R T$, the effect of changing an RT deadline could not be interpreted as simply producing a change in the probabilities defining a mixture of two distributions. However, within an RT deadline, changes in stimulus similarity produced $R T$ results consistent with a two-state mixture theory of discrimination RT. With a fixed RT deadline, latency distributions associated with states of the model remained invariant as stimulus similarity decreased. But changes in the RT deadline produced changes in the latency distributions associated with states of the model. The results suggested that in order to meet the demands of an RT deadline, Ss exert control over their discriminative RT distributions.

Since Ss control their RT distributions in order to meet an E-imposed RT deadline, it is possible for the $E$ to track the temporal development of a perceptual discrimination by imposing RT deadlines on the experimental task. In each RT deadline condition, mean results can be computed so that increases in accuracy performance can be compared across increases in $\mathrm{RT}$ in

*This research was supported by Grant 214-1804 from the National Research Council of Canada and by Grant 214-7804 from the Division of Science and Engineering, McMaster University.

fThe author expresses his appreciation of the contributions made to this project by $A$. D. Tindall, Meri Fish, and D. Ascher. order to examine the trade of speed for accuracy. To obtain adequate data, the $\mathbf{E}$ may find trial-to-trial changes in the RT deadline a convenient means of avoiding statistical difficulties produced by gathering data in blocks of trials where mean RT may be influenced by practice, fatigue, or other systematic effects. But the use of trial-to-trial changes in RT deadlines may introduce a collection of undesired results. For example, a change from one RT deadline to another may not influence $R T$ performance immediately. A series of trials, at the new RT deadline, may be required before a state of responding uninfluenced by the shift in RT deadlines is reached. If so, more complications would be introduced than would be avoided and a careful $E$ would not randomize RT deadlines over the trials of an experimental session. Alternatively, the results obtained by Link \& Tindall (1971) indicate that $S s$ have a much greater degree of control over discrimination RT than had previously been known, and RT control may be sufficient to allow one-trial shifts in performance when RT deadlines are presented at random. Ss may, for example, choose a critical count for a temporally correlated counter and simply respond when the critical count is reached. If several RT deadlines result in $S$ choosing several critical counts, then changes from trial to trial in RT deadlines may simply produce trial-to-trial changes in S's critical count. In the latter case, trial-to-trial changes in RT deadlines would be a convenient method for tracking the speed accuracy tradeoff.

The experiment reported here was designed to determine if there was any influence on mean $R T$ by $R T$ deadlines which were varied from trial to trial. It was found that Ss could immediately shift performance to meet demands of the RT deadline on Trial $n$. No systematic effect of the RT deadline of Trial $n-1$ on the response measures for Trial $n$ was found.

METHOD

Five right-handed university students were each paid $\$ 24.00$ to participate in 12 experimental sessions. A session, lasting approximately $50 \mathrm{~min}$, consisted of two blocks of 250 trials each, separated by a rest period of $5 \mathrm{~min}$. On each trial, $S$ initiated a sequence of trial events by depressing a trial initiation key (TIK). During the depression of TIK, a ready signal, the character $R$, together with an RT deadine in milliseconds were presented for $500 \mathrm{msec}$ on a computer-controlled oscilloscope (Tektronix 602 with P4 phosphor) placed $1 \mathrm{~m}$ in front of the $S$. Immediately following the ready-signal/deadline display, the first of two horizontal line segments was presented for $200 \mathrm{msec}$, followed by an interstimulus interval of $200 \mathrm{msec}$ during which the oscilloscope display screen was blank. The second line segment was presented until $S$ made a "same" or "different" response by releasing TIK and then, with the same hand, depressing one of two (80-g) microswitches. After responding, S could be informed via the oscilloscope whether or not the response was correct (YES or NO) and whether or not the RT deadline was exceeded (SPEED OK or TOO SLOW). Each feedback display lasted 500 msec and was followed by a new trial when $S$ next depressed TIK. If, during any trial, $S$ released TIK before the presentation of the second line segment, the trial was aborted but restarted with the next depression of TIK. The RTs were measured from the onset of the second line segment to the depression of one of the two choice response microswitches.

Five horizontal line segments, 2.0, $1.9,1.8,1.7$, and $1.6 \mathrm{~cm}$, were used throughout the experiment. For three Ss the $2.0-\mathrm{cm}$ line segment was always the standard, and was presented as the first of the two line segments. For the remaining two $\mathrm{Ss}$, the $1.6 \mathrm{-cm}$ line segment was the standard. The differences between the standard and comparison stimuli will be referred to as $0 \Delta S, 1 \Delta S, 2 \Delta S, 3 \Delta S$, and $4 \Delta S$, representing 0 - to $4-\mathrm{mm}$ differences. On each trial, the RT deadline was selected at random from a set of three deadlines: 260 or $460 \mathrm{msec}$, or accuracy (acc). The instructions to $S$ indicated that both the RT deadline and difficulty of the task would vary from trial to trial and that the $S$ was to "beat the time" while being as accurate as possible. For both the 260-msec and the 460-msec deadlines, Ss were given feedback on both speed and accuracy, while in the accuracy case only accuracy feedback was provided, but, in this case, a blank display replaced the usual RT deadline feedback display.

Each session yielded a total of 500 
Table 1

Estimates of the Probability of a Correct Response $\left(P_{c}\right)$, the Mean Correct $R T$ ( $\left.M_{c}\right)$, and the Mean Error RT $\left(M_{e}\right)$ for Each $S$ and Averages Across $S s$

\begin{tabular}{|c|c|c|c|c|c|c|c|c|c|c|}
\hline \multirow[b]{3}{*}{$\mathrm{S}$} & \multirow{3}{*}{$\begin{array}{c}\text { Stimulus } \\
\text { Differ } \\
\text { ence }\end{array}$} & \multicolumn{6}{|c|}{ RT Deadlines } & \multirow{2}{*}{\multicolumn{3}{|c|}{ Accuracy }} \\
\hline & & \multicolumn{3}{|c|}{$260 \mathrm{Msec}$} & \multicolumn{3}{|c|}{$460 \mathrm{Msec}$} & & & \\
\hline & & $\mathbf{P}_{\mathbf{c}}$ & $\mathbf{M}_{\mathrm{c}}$ & $\mathrm{M}_{\mathrm{e}}$ & $\mathbf{P}_{\mathbf{c}}$ & $\mathbf{M}_{\mathbf{C}}$ & $\mathrm{M}_{\mathrm{e}}$ & $\mathbf{P}_{\mathrm{C}}$ & $\mathrm{M}_{\mathrm{c}}$ & $\mathbf{M}_{\mathbf{e}}$ \\
\hline BT & $\begin{array}{l}0 \Delta \\
1 \Delta \\
2 \Delta \\
3 \Delta \\
4 \Delta\end{array}$ & $\begin{array}{l}.519 \\
.545 \\
.685 \\
.675 \\
.585\end{array}$ & $\begin{array}{l}242 \\
224 \\
239 \\
241 \\
244\end{array}$ & $\begin{array}{l}212 \\
214 \\
213 \\
203 \\
192\end{array}$ & $\begin{array}{l}.793 \\
.620 \\
.895 \\
.965 \\
.970\end{array}$ & $\begin{array}{l}399 \\
406 \\
394 \\
382 \\
374\end{array}$ & $\begin{array}{l}340 \\
389 \\
371 \\
276 \\
284\end{array}$ & $\begin{array}{l}.379 \\
.570 \\
.935 \\
.975 \\
.980\end{array}$ & $\begin{array}{l}423 \\
456 \\
422 \\
398 \\
403\end{array}$ & $\begin{array}{l}427 \\
414 \\
373 \\
310 \\
353\end{array}$ \\
\hline IC & $\begin{array}{l}0 \Delta \\
1 \Delta \\
2 \Delta \\
3 \Delta \\
4 \Delta\end{array}$ & $\begin{array}{l}.455 \\
.570 \\
.620 \\
.620 \\
.575\end{array}$ & $\begin{array}{l}227 \\
221 \\
214 \\
246 \\
248\end{array}$ & $\begin{array}{l}215 \\
211 \\
206 \\
199 \\
205\end{array}$ & $\begin{array}{l}.801 \\
.500 \\
.845 \\
.925 \\
.935\end{array}$ & $\begin{array}{l}456 \\
421 \\
414 \\
389 \\
369\end{array}$ & $\begin{array}{l}372 \\
416 \\
315 \\
246 \\
266\end{array}$ & $\begin{array}{r}.801 \\
.740 \\
.935 \\
.995 \\
1.000\end{array}$ & $\begin{array}{l}588 \\
578 \\
483 \\
456 \\
430\end{array}$ & $\begin{array}{c}546 \\
620 \\
442 \\
223 \\
-\end{array}$ \\
\hline SJ & $\begin{array}{l}0 \Delta \\
1 \Delta \\
2 \Delta \\
3 \Delta \\
4 \Delta\end{array}$ & $\begin{array}{l}.524 \\
.545 \\
.460 \\
.470 \\
.435\end{array}$ & $\begin{array}{l}181 \\
194 \\
176 \\
201 \\
189\end{array}$ & $\begin{array}{l}211 \\
173 \\
176 \\
191 \\
172\end{array}$ & $\begin{array}{l}.809 \\
.565 \\
.945 \\
.930 \\
.960\end{array}$ & $\begin{array}{l}452 \\
410 \\
416 \\
377 \\
374\end{array}$ & $\begin{array}{l}360 \\
436 \\
417 \\
371 \\
322\end{array}$ & $\begin{array}{l}.866 \\
.610 \\
.915 \\
.985 \\
.995\end{array}$ & $\begin{array}{l}545 \\
558 \\
509 \\
467 \\
429\end{array}$ & $\begin{array}{l}503 \\
578 \\
553 \\
752 \\
152\end{array}$ \\
\hline $\mathbf{M B}$ & $\begin{array}{l}0 \Delta \\
1 \Delta \\
2 \Delta \\
3 \Delta \\
4 \Delta\end{array}$ & $\begin{array}{l}.480 \\
.555 \\
.550 \\
.605 \\
.615\end{array}$ & $\begin{array}{l}174 \\
175 \\
191 \\
176 \\
198\end{array}$ & $\begin{array}{l}175 \\
171 \\
177 \\
178 \\
166\end{array}$ & $\begin{array}{l}.819 \\
.585 \\
.890 \\
.965 \\
.980\end{array}$ & $\begin{array}{l}414 \\
400 \\
394 \\
393 \\
376\end{array}$ & $\begin{array}{l}338 \\
412 \\
363 \\
352 \\
302\end{array}$ & $\begin{array}{r}.929 \\
.600 \\
.960 \\
.995 \\
1.000\end{array}$ & $\begin{array}{l}538 \\
621 \\
524 \\
466 \\
445\end{array}$ & $\begin{array}{c}627 \\
621 \\
560 \\
990 \\
-\end{array}$ \\
\hline IT & $\begin{array}{l}0 \Delta \\
1 \Delta \\
2 \Delta \\
3 \Delta \\
4 \Delta\end{array}$ & $\begin{array}{l}.339 \\
.595 \\
.675 \\
.715 \\
.645\end{array}$ & $\begin{array}{l}231 \\
220 \\
222 \\
223 \\
220\end{array}$ & $\begin{array}{l}207 \\
240 \\
205 \\
198 \\
213\end{array}$ & $\begin{array}{l}.621 \\
.680 \\
.860 \\
.960 \\
.955\end{array}$ & $\begin{array}{l}388 \\
357 \\
357 \\
350 \\
345\end{array}$ & $\begin{array}{l}319 \\
370 \\
327 \\
329 \\
246\end{array}$ & $\begin{array}{r}.916 \\
.650 \\
.940 \\
1.000 \\
.995\end{array}$ & $\begin{array}{l}566 \\
675 \\
538 \\
496 \\
479\end{array}$ & $\begin{array}{c}681 \\
584 \\
642 \\
- \\
466\end{array}$ \\
\hline Average & $\begin{array}{l}0 \Delta \\
1 \Delta \\
2 \Delta \\
3 \Delta \\
4 \Delta\end{array}$ & $\begin{array}{l}.463 \\
.562 \\
.598 \\
.617 \\
.571\end{array}$ & $\begin{array}{l}210 \\
207 \\
211 \\
219 \\
221\end{array}$ & $\begin{array}{l}204 \\
201 \\
192 \\
193 \\
188\end{array}$ & $\begin{array}{l}.769 \\
.590 \\
.887 \\
.949 \\
.960\end{array}$ & $\begin{array}{l}424 \\
397 \\
396 \\
378 \\
368\end{array}$ & $\begin{array}{l}341 \\
407 \\
348 \\
312 \\
279\end{array}$ & $\begin{array}{l}.878 \\
.634 \\
.937 \\
.990 \\
.994\end{array}$ & $\begin{array}{l}531 \\
580 \\
495 \\
457 \\
437\end{array}$ & $\begin{array}{l}541 \\
556 \\
511 \\
502 \\
338\end{array}$ \\
\hline $\begin{array}{c}\text { Standard } \\
\text { Errors }\end{array}$ & $\begin{array}{l}0 \Delta \\
1 \Delta \\
2 \Delta \\
3 \Delta \\
4 \Delta\end{array}$ & $\begin{array}{l}.008 \\
.016 \\
.016 \\
.015 \\
.016\end{array}$ & & & $\begin{array}{l}.007 \\
.016 \\
.010 \\
.007 \\
.006\end{array}$ & & & $\begin{array}{l}.005 \\
.015 \\
.006 \\
.001 \\
.001\end{array}$ & & \\
\hline
\end{tabular}

observations. The first two sessions were ignored as practice sessions and in the remaining sessions the first 10 trials of a block were treated as practice trials and ignored. Within the 240 remaining trials of each block there were $1200 \Delta \mathrm{S}$ presentations and 30 presentations of each of the remaining four stimulus differences. Since the RT deadline was varied from trial to trial, equiprobably and at random, each $R T$ deadline occurred on 80 trials. Thus, within each block, any combination of a particular RT deadline and $\mathrm{K} \Delta \mathrm{S}(\mathrm{K}=0,1,2,3,4)$ yielded 40 observations for $K=0$ and 10 observations for each of the four remaining values of $K$. Since two blocks constituted an experimental session and since there were 10 sessions and $5 \mathrm{Ss}$, there were 8,000 trials at each of the three RT deadlines split into $4,0000 \Delta \mathrm{S}$ observations and 1,000 observations for each of the stimulus differences greater than 0 . In all, 24,000 observations enter into the analysis of the experiment. RESULTS

Estimates of the marginal reaction time, are not dependent upon the RT deadline of the previous trial. It is particularly impressive that $S s$ faced with a task of responding within a 460-msec RT deadline can shift to the 460-msec deadline from either a 260-msec RT deadline or the acc condition and perform the same as when no change in the RT deadline occurred. Moreoever, the results in Fig. 1 are consistent in terms of correct response probability and mean correct and error response times.

The marginal results for the 460-msec RT deadline trials were expanded so that a detailed study of performances across the five levels of discriminable difference could be compared for cases where the RT deadline on the preceding trial was $260 \mathrm{msec}, 460 \mathrm{msec}$, or acc. These results are shown in Table 2 . It can be seen that within stimulus differences, the maximum range of differences in probability of a correct response is .044 , while between stimulus differences, the maximum range is .397. We can be confident that the major source of variability can be attributed to stimulus differences, while for a particular stimulus difference the RT deadline of the preceding trial has little, if any, effect.

Similar conclusions can be drawn concerning the mean correct RTs where the largest difference in mean RT within stimulus differences is $15 \mathrm{msec}$ (with a standard error of the difference of 10.0), while for between stimulus differences, the maximum difference in mean $R T$ is $58 \mathrm{msec}$ (with a standard error of the difference of $3.2 \mathrm{msec}$ ). Using the Studentized range based on within-S variances, it was concluded that and the mean correct and error response times, $M_{c}$ and $M_{e}$, are given in Table 1. Mean values, computed across Ss and standard errors are presented in the lower part of Table 1. From the average results, it is clear that in two conditions $(460 \mathrm{msec}$ and acc) mean RT varies as a function of $\Delta S$, while for the 260 -msec condition mean $\mathrm{RT}$ remains relatively constant across all values of $\Delta S$. The probability of a correct response is, in general, smallest for a $1 \Delta \mathrm{S}$ discrimination, larger for the $0 \Delta \mathrm{S}$ case, and then increasingly large for 2,3 , and $4 \Delta S$.

The major result from this experiment is pictured in Fig. 1. To obtain these results, the probability of a correct response and the mean correct and error response times were obtained for each RT deadline- $\Delta S$ combination, conditionalized on the RT deadline of the preceding trial. These results were then averaged across values of $\Delta S$ to provide a large number of observations. As can be seen in Fig. 1, shifts in performance, as measured by either probability or

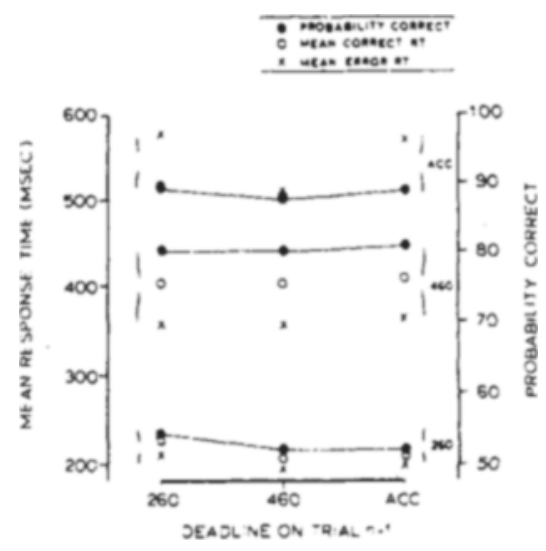

Fig. 1. Within brackets are placed results for each RT deadline on Trial $n$ conditionalized on the RT deadline of Trial $n-1$. Xs are estimates of mean error RT, and filled circles are estimates of correct response probability. Open circles represent estimates of mean correct response time. 
Table 2

Results for the 460-msec RT Deadline Conditionalized Upon the RT Deadline of the Preceding Trial

Estimates

\begin{tabular}{|c|c|c|c|c|c|c|c|c|c|c|c|c|}
\hline \multirow{2}{*}{$\begin{array}{c}\text { Stimulus } \\
\text { Differ- } \\
\text { ence }\end{array}$} & \multicolumn{3}{|c|}{$\mathbf{P}_{\mathbf{c}}$} & \multicolumn{3}{|c|}{$\mathrm{M}_{\mathrm{c}}$ (msec) } & \multicolumn{3}{|c|}{$M_{e}($ msec $)$} & \multicolumn{3}{|c|}{$\mathrm{N}$} \\
\hline & 260 & 460 & Acc & 260 & 460 & Acc & 260 & 460 & Acc & 260 & 460 & Acc \\
\hline $0 \triangle$ & .762 & .773 & .771 & 428 & 425 & 428 & 353 & $\mathbf{3 3 0}$ & 348 & 1337 & 1241 & 1422 \\
\hline $1 \Delta$ & .614 & .581 & .576 & 401 & 393 & 408 & 396 & 415 & 421 & 326 & 384 & 290 \\
\hline $2 \triangle$ & .891 & .892 & .877 & 393 & 398 & 405 & 336 & 360 & 352 & 284 & 399 & 317 \\
\hline $3 \Delta$ & .929 & .943 & .973 & 381 & 379 & 383 & 308 & 292 & 368 & 336 & 298 & $\mathbf{3 6 6}$ \\
\hline \multirow[t]{4}{*}{$4 \Delta$} & .959 & .952 & .970 & 373 & 367 & 372 & 294 & 273 & 273 & 390 & 311 & 299 \\
\hline & \multicolumn{9}{|c|}{ Standard Errors } & & & \\
\hline & \multicolumn{3}{|c|}{$P_{c}$} & \multicolumn{3}{|c|}{$M_{c}$ (msec) } & \multicolumn{3}{|c|}{$\mathbf{M}_{\mathrm{e}}$ (msec) } & & & \\
\hline & 260 & 460 & Acc & 260 & 460 & Acc & 260 & 460 & Acc & & & \\
\hline $0 \triangle$ & .012 & .012 & .012 & 3.4 & 3.7 & 3.4 & 8.4 & 5.4 & 6.6 & & & \\
\hline $1 \Delta$ & .027 & .025 & .029 & 8.2 & 6.6 & 10.0 & 8.2 & 7.7 & 8.7 & & & \\
\hline $2 \Delta$ & .019 & .016 & .018 & 7.8 & 5.6 & 6.5 & 13.5 & 12.8 & 14.4 & & & \\
\hline $3 \Delta$ & .013 & .013 & .010 & 5.2 & 5.6 & 3.9 & 13.7 & 17.9 & 23.4 & & & \\
\hline $4 \Delta$ & .010 & .012 & .010 & 4.2 & 5.7 & 6.0 & 15.6 & 15.8 & 24.7 & & & \\
\hline
\end{tabular}

Note-The number of observations, $N$, relers to the number of transitions from the specified deadline on Trial $n-1$ to a 460-msec deadline on Trial n. The total number of transitions per block of trials will include a transition from Trial 10 to Trial 11 of each block.

differences between mean RTs existed between stimuli, but not across means conditionalized on the preceding trial RT deadlines. Similar results were not obtained for the mean error RTs largely because of small numbers of observations. In one case, only 9 observations were available, and, in another case, only 10 were available. Considering the large number of observations entering into the analyses of correct response probability and mean RT, it appears safe to conclude that response measures on Trial $n$ are not systematically influenced by the RT deadline of the immediately preceding trial.

\section{DISCUSSION}

The major experimental result shown in Fig 1 indicates that trial-to-trial changes in E-imposed RT deadlines are immediately adjusted to by $S$. Whether the RT deadline was changed from $460 \mathrm{msec}$ or acc to $260 \mathrm{msec}$, Ss can perform equally well. This result may not seem surprising given the extremely low mean RT for 260-msec deadline trials. Since $S$ was required first to release a trial initiation (or home) key and then make a choice response by moving his hand approximately $10 \mathrm{~cm}$ forward to depress the choice key, the mean RT of approximately 200 msec is very fast indeed. Were it not for the slight differences in response probability as a function of $\Delta \mathrm{S}$ given in Table 1 , it might be concluded that all responding on the 260-msec deadline trials represented guessing from a state of minimum response time and minimal stimulus information. It appears more likely that performance on the 260 -msec deadline trials is a mixture deadline is imposed on $S$. In this case, if the deadline of a preceding trial was $260 \mathrm{msec}$, then S's RT and acc are both increased. If an acc trial preceded the 460 -msec deadline trial, then both RT and acc decreased. The fact of interest is that from a 260-msec deadline, $S$ pushes performance up to the same level that he pushes his performance down to from an acc trial. Furthermore, the change from a 460-msec deadline to a 460-msec deadline produces results identical to those obtained when the deadline on the prior trial was either $260 \mathrm{msec}$ or acc. This strongly suggests that performance under an RT deadline may be dominated by the temporal constraints of the task and supports conclusions drawn from previous experiments (Link \& Tindall, 1971).

Conclusions similar to those drawn from 260- and 460-msec deadline results can be obtained from acc trial results, although there is a significant compromising result. Since the mean error RT for an acc trial preceded by a 460 -msec deadline is substantially, and significantly, different from other mean error RTs, it is conceivable that
$S$ sometimes fails to shift into a mode of responding determined by only maximal acc. If so, the argument that RT deadlines produce S-controlled RT distributions is not damaged, since the inability to switch from a $460-\mathrm{msec}$ deadline trial to unrestricted RT responding implies that $R T$ is somehow controlled on the preceding 460-msec deadline trial. Finally, measures of correct response probability and mean $\mathrm{RT}$ are not affected by the deadline of the preceding trial. Thus even results from acc trials support the notion that RT deadlines on Trial $n-1$ have little, if any, influence on performance on Trial $\mathbf{n}$.

From the results shown in Fig. 1, together with the results in Table 2 , we may conclude that the presentation of an RT deadline has an immediate effect and is sufficient to promote a marked change in S's responding. Naturally it is the nature of the change that is most interesting.

The marginal results in Table 1 provide corroborative evidence for two different views of discrimination RT performance. In particular, the marginal probabilities indicate that across stimulus difference greater than zero there exists a virtually constant difference between correct response probability on 460-msec and acc trials. These data are shown in Fig. 2 and support a similar finding (also shown in Fig. 2) reported earlier (Link \& Tindall, 1971). Although differences in the level of responding may depend upon Ss, experimental design, stimulus differences, and RT deadlines, the differences between measures of

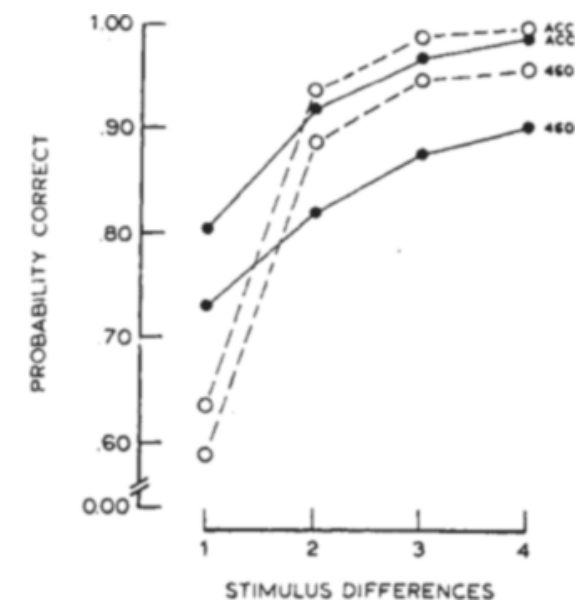

Fig. 2. Estimates of correct response probability across stimulus differences for each of two RT conditions in two similar experiments. Filled circles represent results from Link \& Tindall (1971); open circles represent results from the present experiment. 
probability of a correct response are not influenced by these factors.

The interpretation of the result depends upon differences in probability correct between stimulus differences. For example, the difference between correct response probabilities for $1 \Delta \mathrm{S}$ and $2 \Delta \mathrm{S}$ stimulus differences on a 460 -msec deadline trial is the same as the difference on an acc trial, although the value of probability correct on acc trials exceeds that of the $460-\mathrm{msec}$ deadline trials. Consequently, the constant difference between correct response probabilities for any two stimuli exists long before asymptotic responding is reached.

In a sense, this result argues in favor of a view of discrimination RT similar to the sequential sampling scheme for choice RT proposed by Laming (1968). In Laming's theory, S places constraints on Kullback's (1959) discrimination information statistic, so that over time the information statistic, in performing a random walk on the dimension of posteriori choice probabilities, eventually reaches one of two choice probability bounds. $S$ then makes the choice response corresponding to the boundary reached by the discrimination information statistic. In the present experiment, where $S$ judges stimuli to be either the same as or different from a standard, only two boundaries are necessary to determine the choice response. As increasingly stringent RT deadlines are placed on $S$, a constant reduction in correct response probability could occur by a simple change in the boundary set for the information statistic. In addition, $S$ would need no more than one pair of boundaries for each RT deadline in order to perform adequately under trial-to-trial changes in RT deadlines.
Although the results are not incompatible with a form of sequential analysis RT theory, neither are they incompatible with a simple alarm clock model of RT. In this theory, it would be assumed that $S$ controls a parameter of his RT distribution so that only a small number of long RTs exceed the RT deadline. By using only three parameters, $S$ could perform trial-to-trial shifts in RT distribution and thus meet the RT deadline constraints. However, the nature of the decision theory needed to account for constant increases in correct response probability as the RT deadline changes from $460 \mathrm{msec}$ to acc is unknown.

It has been suggested by Audley \& Mercer (1968), Thomas (1971), and others that discrimination $\mathrm{RT}$ is related to the absolute distance of an evoked sensory representation of a stimulus from a S-controlled decision criterion. Assuming the usual signal detection model, the assumption is simply that the nearer a sensory value is to the S's decision criterion, the more difficult the decision and (consequently?) the longer the RT. If, in the present experiment, we assume that for any fixed RT deadline there exists a single sensory distribution for the standard and a series of sensory distributions for the comparison stimuli, and a single decision criterion, then the response "same," given a difference between the standard and comparison, can be thought of as a miss. According to the Audley and Mercer and the Thomas notion, median RT for misses should increase as the probability of a miss decreases. The results from the present experiment do not confirm this rather general prediction.

In summary, it has been shown that trial-to-trial changes in RT deadlines can be tracked by the $S$ and that the influence of the RT deadline used in Trial $n-1$ on the S's performance on Trial $n$ is, if existent, minimal. The marginal probability results indicate that $S$ could operate in a manner consistent with Laming's (1968) theory for choice RT. But the data are also consistent with an alarm clock view of RT control. Future experiments designated to distinguish between the different views of discrimination RT can benefit from the empirical results that, on a trial-to-trial basis, $S s$ can alter performance to meet the demands of randomly presented $\mathrm{RT}$ deadlines.

\section{REFERENCES}

AUDLEY, R J \& MERCER, A. The relation between decision time and relative response frequency in blue-green discriminations. British Journal of Mathematical \& Statistical Psychology, $1968,21,183-192$.

FITTS, P. M. Cognitive aspects of information processing: III. Set for speed versus accuracy. Journal of Experimental Psychology, $1966,71,849-857$.

KULLBACK, S. Information theory and statistics. New York: Wiley, 1959

LAMING, D. Information theory of choice reaction time. New York: Academic Press, 1968.

LINK, S. W., \& TINDALL, A. D. Speed and accuracy in comparative judgments of line length. Perception \& Psychophysics, $1971,9,284-288$

PACHELLA, R. A., \& PEW, R. W. Effect of discrete criterion times. Joumal of Experimental Psychology, 1968, 76, 19-24.

THOMAS, E. A. C. Sufficient conditions for monotone hazard rate. An application to latency-probability curves. Journal of Mathematical Psychology, 1971, 8, 303-332.

YELLOTT, J. 1. Correction for guessing in choice reaction time. Psychonomic Science, 1967, 8, 321-322.

YELLOTT, J. I. Correction for guessing and the speed-accuracy tradeoff in choice reaction time. Journal of Mathematical Psychology, 1971, 8, 159-199. 